\section{Inflammation regulates long non-coding RNA-PTTG1-1:1 in myeloid leukemia}

Elevated tumor necrosis factor (TNF) $\alpha$ serum levels constitute an adverse prognostic factor for the survival of acute myeloid leukemia (AML) patients. ${ }^{1}$ TNF $\alpha$ is produced by various immune effectors and directly or indirectly acts as a pro-proliferative autocrine tumor growth factor. $^{2}$

TNF $\alpha$-controlled regulatory mechanisms especially by long non-coding (lnc)RNA largely remain to be investigated. Here we used DNA microarrays, validated by RNA sequencing, to determine most regulated coding and non-coding transcripts after TNF $\alpha$ stimulation of erythroleukemic TF-1 AML cells. We selected the IncRNA PTTG1-1:1, also identified as MIR3142HG or ENST00000517927, as the most upregulated IncRNA. This long intergenic non-coding RNA of 2301 bases comprises two exons, is strongly conserved in higher vertebrates (Online Supplementary Figure S1) and co-expressed with genes associated with the inflammatory response (Online Supplementary Figure S2). It has recently been reported to be downregulated in adrenocortical carcinoma. ${ }^{3}$ We investigated its regulation by pro-inflammatory mediators and validated differential expression in AML cell lines and patient samples compared to healthy controls (Online Supplementary Figure S3). We validated the translational impact of expression of PTTG1-1:1 by in silico analysis using BloodSpot BloodPool, ${ }^{4}$ The Cancer Genome Atlas (TCGA), Verhaak and Therapeutically Applicable Research to Generate Effective Treatment (TARGET) AML databases.

In line with a role in inflammation, the putative promoter region of PTTG1-1:1 surrounding the transcriptional start site (TSS) from $-2,000$ to +500 base pairs (bp) (Online Supplementary Figure S1) revealed two nuclear factor $(\mathrm{NF})-\mathrm{\kappa B}$ binding sites within a chromatin area hypersensitive to DNase I characterized by elevated histone (H)3K27 acetylation (Ac) and H3K4 monomethylation (Me1) and trimethylation (Me3) levels, reflecting transactivation potential. None of the predicted peptides (over
A

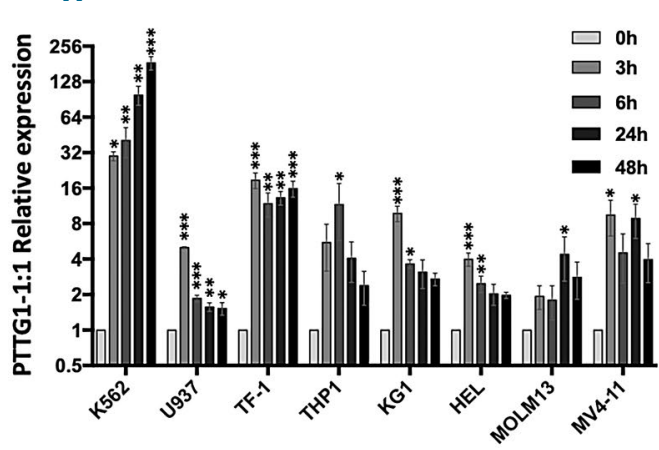

B
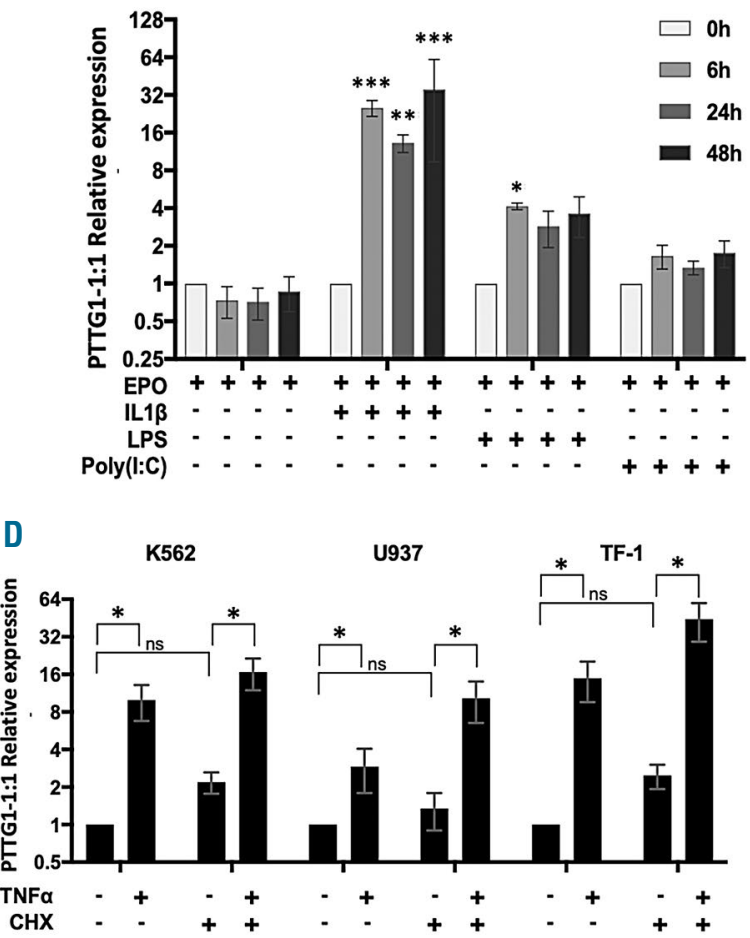

E

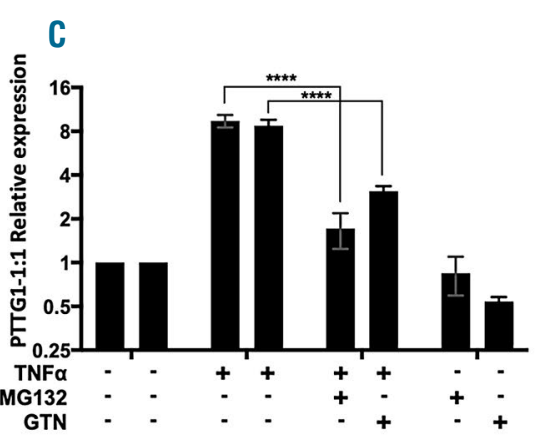

miR-146a
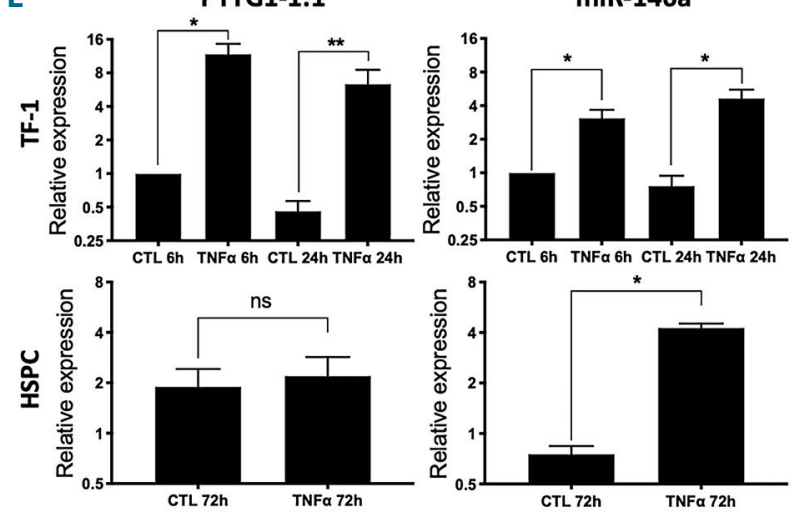

Figure 1. Regulation of PTTG1-1:1 expression by inflammatory mediators. (A) TNF $\alpha$-induced PTTG1-1:1 expression in leukemia cell lines. (B) PTTG1-1:1 expression in TF-1 cells induced by pro-inflammatory mediators: IL-1 $\beta$, LPS and poly(I:C) after 6,24 and 48 hours (h). (C) Effect of NF-kB inhibition by MG132 or goniothalamin (GTN) on TNF $\alpha$-induced PTTG1-1:1 expression in TF-1 cells. (D) Effect of protein synthesis inhibition by cycloheximide (CHX) on TNF $\alpha$-induced PTTG1-1:1 expression. (E) Relative expression of PTTG1-1:1 and miR-146a in TF-1 cells and hematopoietic stem progenitor cells (HSPC) after 6, 24 or 72 h. Expression levels are indicated relative to $\beta$-actin and the basal expression level in untreated controls. Data were expressed as the average \pm standard error of the mean (SEM). Significance: ${ }^{\star} P<0.05 ; * * P<0.01 ; * * * P<0.001$; $* * * * P<0.0001$. 
10 amino acids) or proteins, that could derive from the putative small open reading frames of the PTTG1-1:1 transcript, were detected in the Protein Family (PFAM) 30.0 mass spectrum data set (Online Supplementary Table S1).

Basal expression levels of PTTG1-1:1 differed in healthy hematopoietic stem progenitor cells (HSPC) from leukemia or solid tumor cell lines (Online Supplementary Figure S3). Inflammatory mediators like TNFa (Figure
1A), interleukin (IL)-1 $\beta$, lipopolysaccharide (LPS) or polyinosinic-polycytidylic acid (poly [I:C]) (Figure $1 \mathrm{~B}$ and Online Supplementary Figure S4) induced a significant celltype- and time-dependent increase of PTTG1-1:1 expression. Real-time (RT)-PCR combined with fluorescence in situ hybridization (FISH) confirmed the cytoplasmic localization of PTTG1-1:1 (Online Supplementary Figure S5A-B and Online Supplementary Table S2). To validate involvement of NF-אB in PTTG1-1:1 regulation, cells pretreated
A

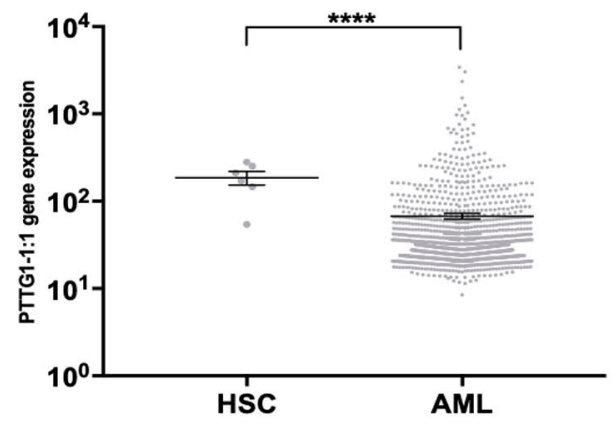

C

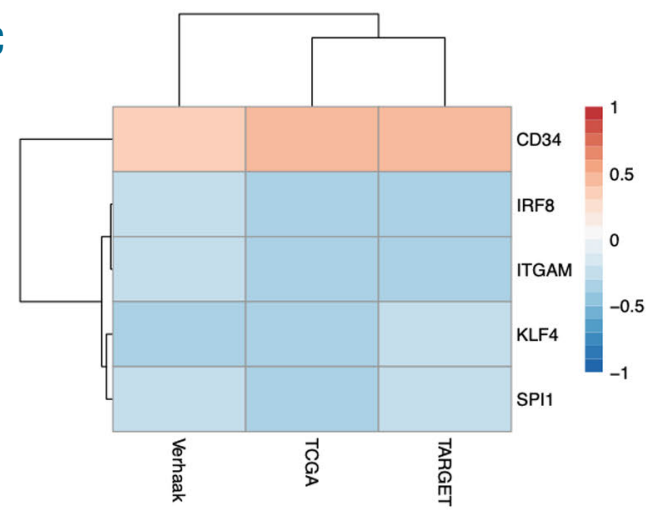

B
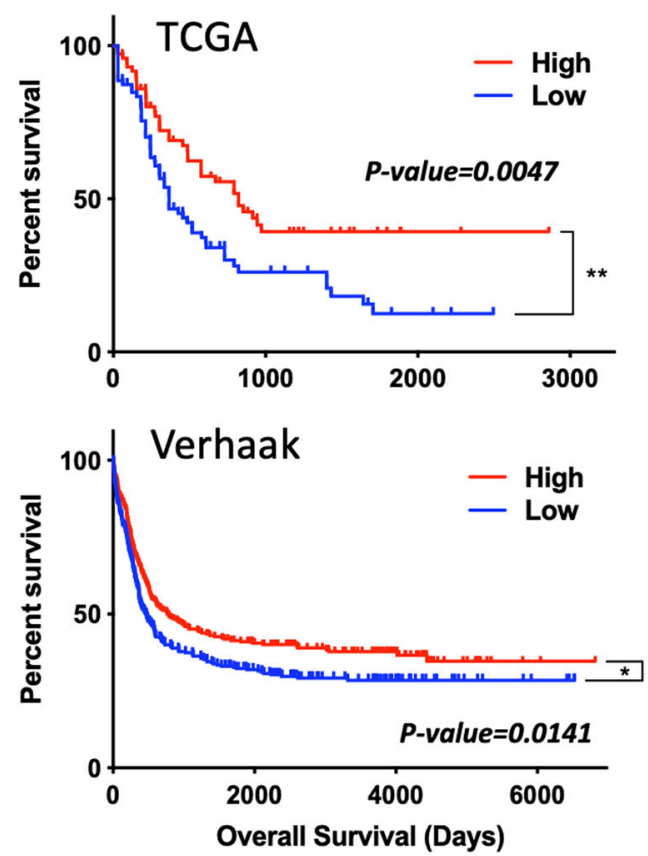

D
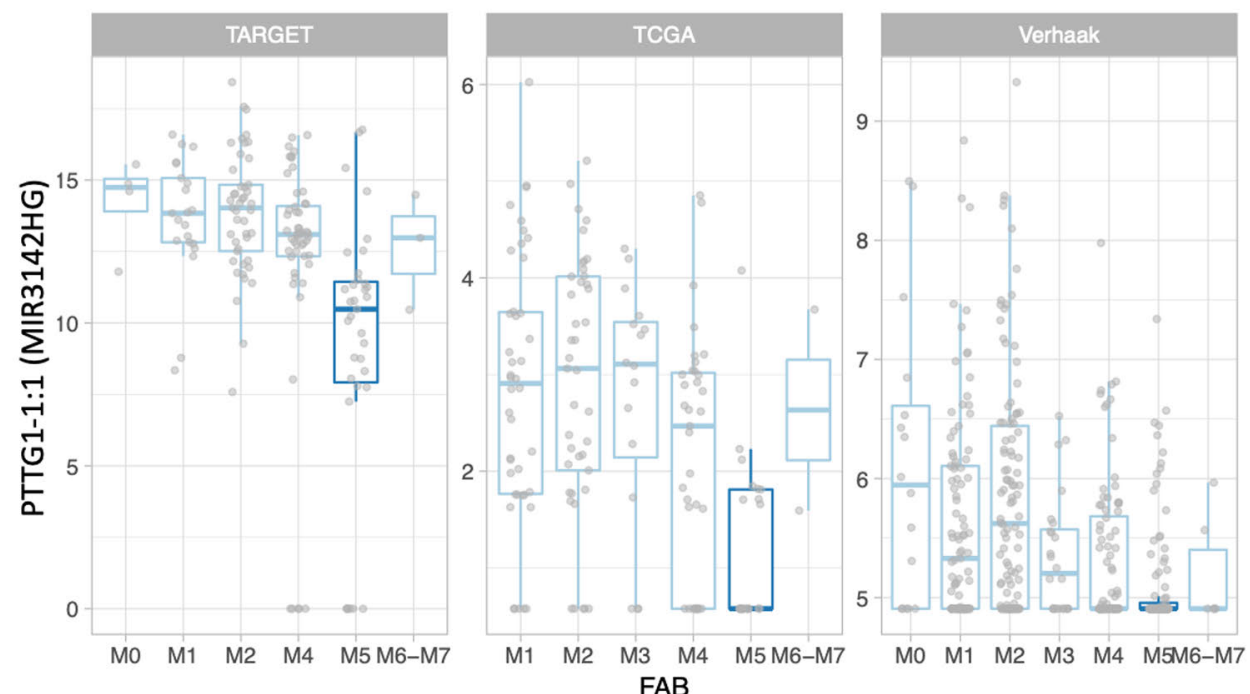

$\mathrm{FAB}$

Figure 2. PTTG1-1:1 expression in primary cells and acute myeloid leukemia patients. (A) BloodPool transcriptomic analysis of LOC285628 (PTTG1-1:1) in acute myeloid leukemia (AML) cells compared to healthy hematopoietic stem cells (HSC). P-values were determined by the Mann-Whitney test. (B) Kaplan-Meier survival curve of AML patients from the TCGA and Verhaak cohorts expressing LOC285628 (PTTG1-1:1) at highest (red, $n=89$ and $=246$ respectively) or lowest levels (blue, $n=89$ and $=275$ respectively). Patients were segregated at the median of expression. (C) PTTG1-1:1 expression was correlated to stemness factor CD34 and inversely correlated to differentiation factors. Heatmap showing Pearson correlation represented as a color, in three public AML patient expression datasets. (D) PTTG1-1:1 expression was lower in M5 AML (in TARGET, TCGA and Verhaak datasets). Data were expressed as the average \pm standard error of the mean (SEM). Significance: $* P<0.05 ; * * P<0.01 ; * * * P<0.001 ; * * * * P<0.0001$. 

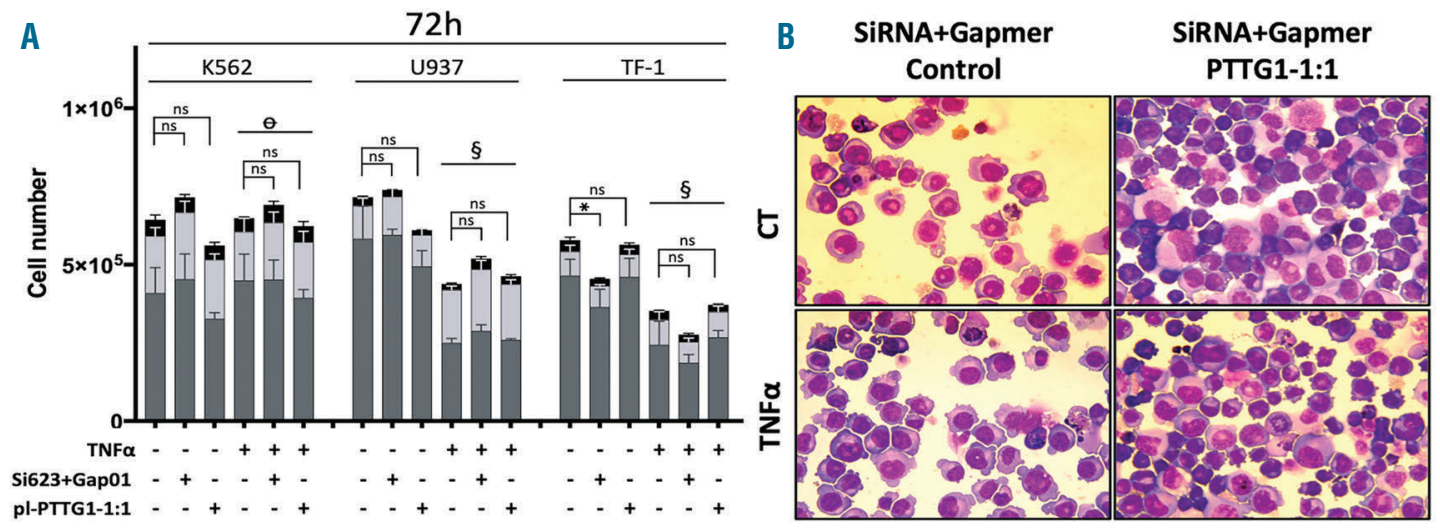

C
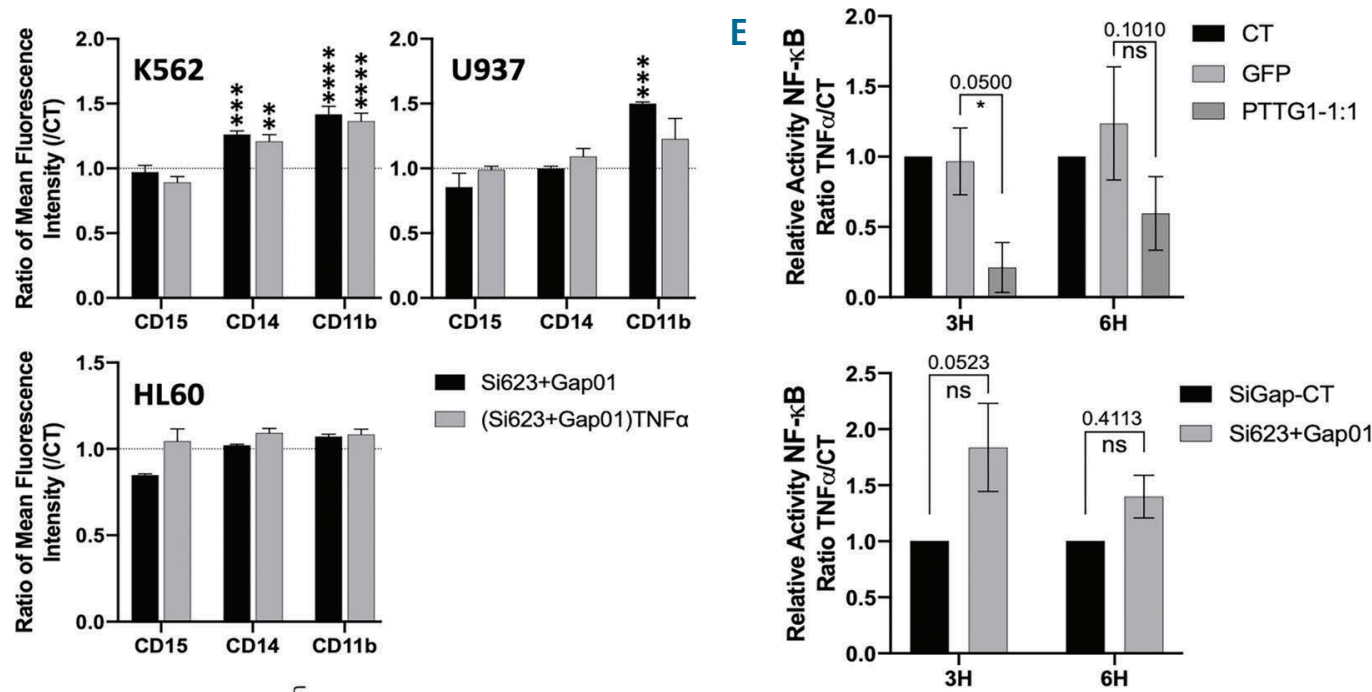

D $\quad 2.0$

Time of treatment with TNFo

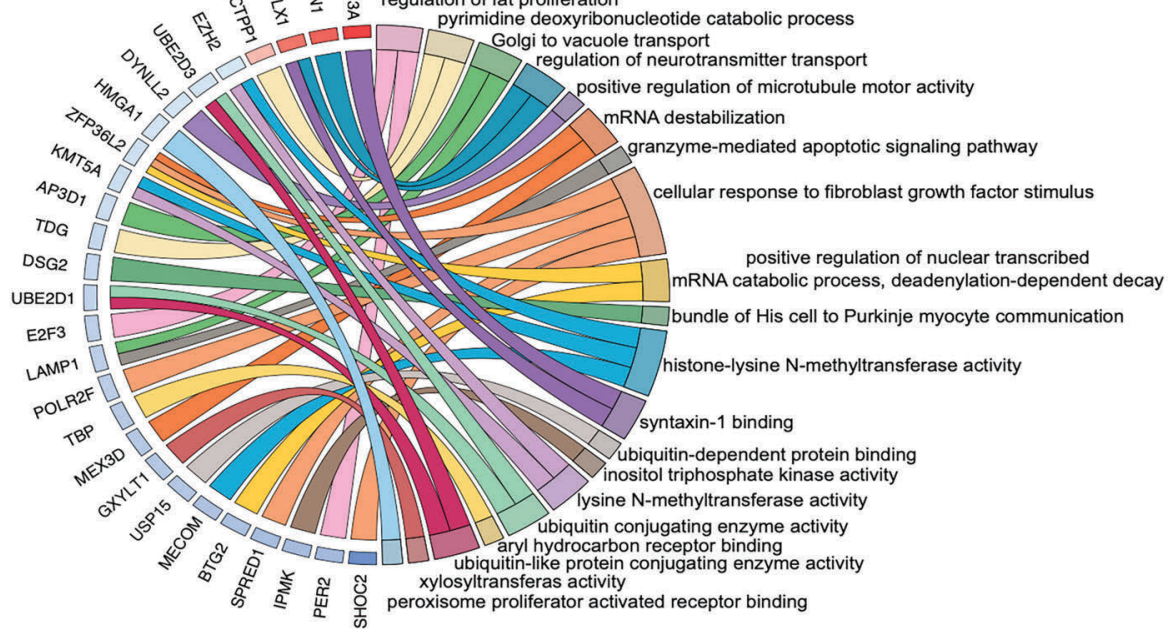

Figure 3. Biological impact of PTTG1-1:1 modulation. (A) Cell viability by Trypan blue exclusion and MitoTracker Red assays (dying cells present a lower mitochondrial membrane potential) in cells transfected with Si623+Gap01 or pIPTTG1-1:1 with or without TNF $\alpha$ treatment. Statistical analyses were performed on living cells, comparing transfected cells to the corresponding controls (without TNF $\alpha$ for Si623+Gap01 and pl-PTTG1-1:1; with TNF $\alpha$ for Si623+Gap01+TNF $\alpha$ and pl-PTTG1-1:1+TNF $\alpha$ ). ns: not significant; ${ }^{*} P<0.05$. Moreover, results show a significant overall effect of TNF $\alpha$ on U937 and TF1 cells $\left({ }^{\circ}\right.$ : not significant; §:P<0.05). (B) K562 cell morphology assessed by May-Grünwald Giemsa (MGG staining), after four days of PTTG1-1:1 knockdown using siRNA and GapmeR. The results show an increase of the average size of cells with PTTG1-1:1 knockdown. (C) Bar plot representing the relative amounts of CD11b, CD14 and CD15 markers in K562, U937 and HL60 cells transfected with (Si623+Gap01) or (SiCt+GapA) with or without TNFa treatment. Data are expressed as the ratio of (SiRNA623+GapA)/(SiCT+GapA) normalized to isotypic controls. (D) Enrichment analysis of significant differentially expressed genes following PTTG1-1:1 knockdown in K562 cells treated with TNF $\alpha$ using the GOplot R package. Depicted genes are connected to their biological or molecular functions through colored ribbons. The outer circle represents log2FC of upregulated (red) or downregulated (blue) genes. (E) NF-KB activity was measured in K562 cells over-expressing PTTG1-1:1 or after PTTG1-1:1 knockdown. Cells were transfected with pl-PTTG1-1:1 expression construct or Si623+Gap01 (targeting PTTG1-1:1) together with the NF-KB luciferase reporter plasmid. P-values from ANOVA are indicated. Data were expressed as the average \pm standard error of the mean (SEM). Significance: $* P<0.05 ; * * P<0.01 ; * * * P<0.001 ; * * * * P<0.0001$ 
with NF-кB inhibitors (proteasome inhibitor MG132 and natural compound goniothalamin $(\mathrm{GTN}))^{5}$ significantly abrogated TNF $\alpha$-induced expression of PTTG1-1:1 in all cell types. Interestingly, in U937 AML cells, basal expression was also significantly abrogated by MG132 (Figure $1 \mathrm{C}$ and Online Supplementary Figure S6A). SiRNA-mediated knockdown of p65/NF- $\mathrm{kB}$ further confirmed involvement of this transcription factor in the regulation of PTTG1-1:1 expression in K562 cells without altering the expression of NF- $\mathrm{BB}$-independent genes SP1 and SP3 (Online Supplementary Figure S6B). Cycloheximide pretreatment showed independency of PTTG1-1:1 regulation from de novo protein synthesis (Figure 1D). RNA sequencing (RNAseq) allowed identification of AML-relevant pathways significantly modulated by PTTG1-1:1 knockdown (Online Supplementary Figure S7). We observed downregulation of heme metabolism, mTOR complex (mTorc)-1 signaling and IL-2-signal transducer and activator of transcription (STAT) 5 signaling and upregulation of the interferon (IFN) $\alpha$ response (Online Supplementary Table S3).

The intron of PTTG1-1:1 encodes the unknown miR3142 whereas its second exon encodes miR-146a (Online Supplementary Figure S1) associated with TNF $\alpha$-mediated induction of IRAK-1 (TNF $\alpha$ and Toll-like receptors (TLR)/IL-1 receptor signaling intermediates IL-1 receptorassociated kinase) and TRAF (TNF receptor-associated factor) 6 , both regulating NF- $\mathrm{B}$ signaling. Mir-3142 could not be detected in any of our samples. Interestingly, our results showed differential expression levels of PTTG1-1:1 and miR-146a in TF-1 and HSPC under inflammatory conditions (Figure 1E), underlining the independent regulation of both ncRNA.

To confirm the involvement of PTTG1-1:1 in myeloid pathologies, we compared the expression of PTTG1-1:1 in HSPC, AML cell lines and 14 AML patients representing different classification and genetic backgrounds. This limited set of patients did not allow to determine a significant expression change (Online Supplementary Figure $S 8 A)$. To further investigate potential changes of PTTG11:1 expression, the BloodPool AML database 4 (Online Supplementary Table S4) allowed us to demonstrate a 2.8fold decrease of its expression (Figure 2A and Online Supplementary Figure S8B). Results are in agreement with the survival curves of the TCGA and Verhaak AML patient cohorts, showing a better survival rate for patients expressing higher levels of PTTG1-1:1 (Figure 2B). The multivariable analysis performed on the TCGA cohort confirmed the impact of PTTG1-1:1 expression on patient survival (Online Supplementary Figure S9). By using public TCGA, Verhaak and the pediatric cohort of the TARGET AML patient datasets (Online Supplementary Tables S5-7), we confirmed that low expression of the PTTG1-1:1 was systematically associated with monocytic transition (decrease of stemness factor CD34 and increase of monocyte factors such as CD11b [integrin $\alpha$ $M$ (ITGAM)], transcription factors Krüppel-like factor (KLF) 4, PU-1 (SPI-1) and interferon regulatory factor (IRF) 8, in the three datasets (Figure 2C). PTTG1-1:1 expression was consistently lower in M5 (acute monocytic leukemia) compared to undifferentiated M0/M1 AML (Figure 2D). Finally, we determined the origin of the measured signal for PTTG1-1:1 or miRNA-146a in the Verhaak Affymetrix dataset or in the RNAseq data from Ensembl. We validated in the different cohorts that the expression measured corresponded to gene sequences different from those of pri/pre-miRNA.

To validate the implication of PTTG1-1:1 in hematopoietic differentiation, we inhibited its expression by a double knockdown approach using LNA ${ }^{\mathrm{TM}}$ GapmeRs and Stealth RNAi ${ }^{\mathrm{TM}}$ siRNA (Si623+Gap01) decreasing the expression by $25 \%$ and $70 \%$ in the absence and presence of $\mathrm{TNF} \alpha$, respectively (Online Supplementary Figure S10). These results were validated by RNAseq (Online Supplementary Figure S15). Inhibition of PTTG1-1:1 did not affect the cell cycle nor cell survival (Figure $3 \mathrm{~A}$ ) and induced a phenotypic modification corresponding to the appearance of a sub-population of cells with increased volume (average increase: 68\%) representing $15.4 \pm 7.4 \%$ of the population (Figure $3 \mathrm{~B}$ and Online Supplementary Figure S11) and an increased level of CD14 and CD11b markers (Figure 3C) corresponding to a commitment of cells towards the monocytic lineage in K562 and U937 cells, whereas HL60 cells showed no phenotypic change. These observations are consistent with data extracted from the GSE42519 dataset, ${ }^{6}$ which shows a progressive decrease of PTTG1-1:1 in hematopoietic cells throughout myeloid differentiation (Online Supplementary Figure S12).

We then cloned PTTG1-1:1 (pl-lncPTTG1-1:1) and transiently overexpressed it in K562 cells. Proliferation, cell cycle distribution and viability remained unchanged (Figure 3A and Online Supplementary Figure S13A-B) compared to mock-transfected cells. However, the overexpression of PTTG1-1:1 significantly downregulated CD15 expression in K562 cells and CD11b and CD15 expression in HL60 cells after five days (Online Supplementary Figure S13C) confirming the involvement of PTTG1-1:1 in the myelomonocytic commitment.

New therapeutic strategies aim to target expression of RNA and specially lncRNA in leukemia but so far, only a few IncRNA have been directly associated with survival of AML patients. In 215 intermediate risk-AML patients, increased expression of HOTAIRM1 was associated with shorter overall survival, shorter leukemia-free survival and a higher cumulative incidence of relapse ${ }^{7}$ whereas increased MALAT1 expression was associated with poor prognosis in M5 patients. ${ }^{8}$

PTTG1-1:1 is regulated by inflammatory pathways with an involvement in myelocytic commitment. Our RNAseq results in PTTG1-1:1 knockdown K562 cells allowed the identification of the most impacted genes (Online Supplementary Figure S14 and Online Supplementary Table S9-10) and the gene ontology (GO) data associated with the top 10 molecular functions and biological processes (Online Supplementary Figure S15A and Figure $3 \mathrm{D}$ ), highlighting a significant impact of PTTG1-1:1 on pathways controlling RNA stabilization/processing and protein maturation/stability.

One of the most interesting findings is the apparent contradictory expression of PTTG1-1:1. Whereas inflammation is considered to be a negative factor in AML, PTTG1-1:1 is induced by inflammatory mediators but its overexpression is correlated with improved patient survival. This observation led to the hypothesis that PTTG1$1: 1$ could be involved in a feedback loop able to interrupt NF- $\mathrm{KB}$ signaling similarly to miR-146a described to inhibit IRAK1 and TRAF6 expression. As PTTG1-1:1 is the host gene for miR-146a yet presents a differential expression pattern, this IncRNA could also be involved in the regulation of inflammatory processes and thus avoid over-activation of NF- $\kappa B$. ${ }^{9}$ Our data confirm this hypothesis as over-expression of PTTG1-1:1 decreases NF- $\kappa B$ activity (Figure 3E).

Interestingly, a separate in silico analysis identified insulin-like growth factor 2 mRNA-binding proteins (IGF2BP) $1,-2$ and -3 , fused in sarcoma/translocated in liposarcoma (FUS/TLS) and human antigen $\mathrm{R}(\mathrm{HuR})$ as 
putative PTTG1-1:1 interaction partners (Online Supplementary Figure S15B). Their associated GO terms confirmed the impact of PTTG1-1:1 on mRNA/siRNA stabilization, processing and interaction. HuR is known to stabilize the 3' untranslated regions of mRNA encoding cytokines IL-2 and IL-3. ${ }^{10}$

Accordingly, PTTG1-1:1 (over)expression could play a dual role by (a) re-establishing physiological levels of proinflammatory NF- $\kappa \mathrm{B}$ activity via a negative feedback loop (Figure 3E) and (b) potentially by stabilizing mRNA via IncRNA binding proteins to preserve the initial proinflammatory signal as PTTG1-1:1 knockdown downregulated IL-2/Stat5 signaling (Online Supplementary Figure S7).

Altogether, our observations demonstrate the potentializing effect of PTTG1-1:1 on the expression of the pleiotropic cytokine IL-2 and its signaling pathway and highlights its dual role in inflammation control via NF- $\kappa B$ retro-control.

Sébastien Chateauvieux, ${ }^{1,2}$ Anthoula Gaigneaux, ${ }^{10}$

Déborah Gérard, ${ }^{1}$ Marion Orsini, ${ }^{1}$ Franck Morceau, ${ }^{1}$

Barbora Orlikova-Boyer, ${ }^{1,2}$ Thomas Farge, ${ }^{3,4}$

Christian Récher, ${ }^{3,4,5}$ Jean-Emmanuel Sarry, ${ }^{3,4}$ Mario Dicato and Marc Diederich

${ }^{\circ}$ Current address: University of Luxembourg, Faculty of Science, Technology and Communication, Life Science Research Unit, Belvaux, Luxemburg.

'Laboratoire de Biologie Moléculaire et Cellulaire du Cancer, Hôpital Kirchberg, Luxembourg, Luxembourg; ${ }^{2}$ College of Pharmacy, Seoul

National University, Gwanak-gu, Seoul, Korea; ${ }^{3}$ Cancer Research Center of Toulouse, UMR 1037 INSERM/ Université Toulouse III-

Paul Sabatier, Toulouse, France; ${ }^{4}$ Université Toulouse III Paul Sabatier,

Toulouse, France and 'Service d'Hématologie, Centre Hospitalier

Universitaire de Toulouse, Institut Universitaire du Cancer de Toulouse

Oncopôle, Toulouse, France

Correspondence: MARCDIEDERICH - marcdiederich@snu.ac.kr doi:10.3324/haematol.2019.217281

Funding: SC, MO, DG, BOB and AG are supported by Télévie PhD grants. Research at LBMCC is funded by the Fondation "Recherche Cancer et Sang", RSL asbl, "Een Haerz fir kriibskrank Kanner" asbl, Télévie Luxembourg and the Fondation "Josée Wolter-Hirtt". Research at SNU is supported by National Research Foundation (NRF) [Grant Number 019R1A2C1009231] and by a grant from the MEST of Korea for Tumor Microenvironment Global Core Research Center (GCRC) [Grant Number 2011-0030001].
Support from Brain Korea (BK21) PLUS program and CreativePioneering Researchers Program at Seoul National University [Funding number: 370C-20160062] are acknowledged.

Acknowledgments: the authors thank Prof. Véronique De Mas and Eric Delabesse for the management of the Biobank BRC-HIMIP (Biological Resources Centre-INSERM Midi-Pyrénées "Cytothèque des hémopathies malignes"), supported by CAPTOR (Cancer Pharmacology of Toulouse-Oncopole and Région) as well as Dr. PJM Valk (Department of Hematology, Erasmus University Medical Center, Rotterdam, Netherlands) for sharing the clinical data associated to GSE6891.

Information on authorship, contributions, and financial \& other disclosures was provided by the authors and is available with the online version of this article at www. haematologica.org.

\section{References}

1. Tian $\mathrm{T}$, Wang M, Ma D. TNF-alpha, a good or bad factor in hematological diseases? Stem Cell Investig. 2014;1:12.

2. Volk A, Li J, Xin J, et al. Co-inhibition of NF-kappaB and JNK is synergistic in TNF-expressing human AML. J Exp Med. 2014;211(6):1093-1108.

3. Koduru SV, Leberfinger AN, Ravnic DJ. Small non-coding RNA abundance in adrenocortical carcinoma: a footprint of a rare cancer. Genomics. 2017;5:99-118.

4. Bagger FO, Sasivarevic D, Sohi SH, et al. BloodSpot: a database of gene expression profiles and transcriptional programs for healthy and malignant haematopoiesis. Nucleic Acids Res. 2016;44(D1):D917-924.

5. Orlikova B, Schumacher M, Juncker T, et al. Styryl-lactone goniothalamin inhibits TNF-alpha-induced NF-kappaB activation. Food Chem Toxicol. 2013;59:572-578

6. Rapin N, Bagger FO, Jendholm J, et al. Comparing cancer vs normal gene expression profiles identifies new disease entities and common transcriptional programs in AML patients. Blood. 2014;123(6):894904

7. Diaz-Beya M, Brunet S, Nomdedeu J, et al. The lincRNA HOTAIRM1, located in the HOXA genomic region, is expressed in acute myeloid leukemia, impacts prognosis in patients in the intermediate-risk cytogenetic category, and is associated with a distinctive microRNA signature. Oncotarget. 2015;6(31):31613-31627.

8. Huang JL, Liu W, Tian LH, et al. Upregulation of long non-coding RNA MALAT-1 confers poor prognosis and influences cell proliferation and apoptosis in acute monocytic leukemia. Oncol Rep. 2017;38(3):1353-1362.

9. Spinello I, Quaranta MT, Riccioni R, et al. MicroRNA-146a and AMD3100, two ways to control CXCR4 expression in acute myeloid leukemias. Blood Cancer J. 2011;1(6):e26

10. Wang JG, Collinge M, Ramgolam V, et al. LFA-1-dependent HuR nuclear export and cytokine mRNA stabilization in T cell activation. J Immunol. 2006;176(4):2105-2113. 